\title{
H4H - Hydration for Health
}

\author{
Max Lafontan \\ Inserm/UPS UMR 1048 - Institut des Maladies Métaboliques et Cardiovasculaires, BP84225, \\ 31432 Toulouse Cedex 4, France
}

A great health concern in many countries is the rising progression of overweight and obesity in societies undergoing nutritional and epidemiological transitions. Overweight and obesity has become a global pandemic that has major health and economic consequences. The global transition in food consumption and in physical activity habits probably plays an important role as discussed by various authors in numerous countries [1,2]. A number of factors, including nutrition and eating behaviours, physical activity profile, psychological influences, environmental determinants as well as genetic and epigenetic cues, require consideration.

Concerning nutritional considerations related to obesity, the health impact of fluid intake is commonly neglected, and fluid intake advices are missing in most of the food pyramids offered to the public. Water requirements and hydration challenges are often neglected since it is considered that methodological bias exist for measurement of water intake or water losses [3]. Nevertheless, over the last decades attention has been focused upon health risks related to overconsumption of sugar-sweetened beverages (SSBs). They constitute a large percentage of energy consumed by youth. Despite the strong resistance from the beverage industry and claims of scientists under their control, recent epidemiologic studies have associated the consumption of SSBs with increased risk of obesity, metabolic alterations and risk of cardiovascular diseases. The cumulative evidence from observational studies and experimental trials pinpoint that regular consumption of SSBs contributes to excess weight gain [4, 5]. The health effects of SSBs consumption remain the subject of intense and controversial scientific debate and utilisation of tangential arguments to make diversion from the centre of the debate is used. Guidelines for overweight and obesity usually include recommendations on changes in physical activity and diet while little or no advice is offered on the importance of hydration practices. Recently recommendations for healthier hydration in obesity and type 2 diabetes prevention have been published [6]. Delineation of global health benefits of a healthier hydration merit more attention. With regard to the trend of increasing SSB 
consumption all over the world, it is of major importance to establish healthier hydration behaviours that might play a role in curbing the curve of obesity progression.

Aside from weight-related questions, there is also a relationship been between hydration and kidney function. Data from observational studies suggest that chronic low fluid intake may be an important factor in the pathogenesis of kidney diseases [7]. It was observed that adults with higher fluid intake are less prone to chronic kidney diseases while chronic kidney diseases were associated with low intake of plain water. Inadequate fluid intake is commonly observed in older adults. More research is needed to study the various impacts of hydration in older adults.

The 5th Annual Scientific Conference of the Hydration for Health Initiative which was organised within the long-term programme of Hydration for Health Meetings and sponsored by Danone Waters and Danone Research was held in Evian on July 3, 2013. Three major topics were considered during this meeting:

1) The first session was devoted to the definition of hydration and delineation of optimal hydration biomarkers.

2) A second session gave an overview of the relationships between hydration status, water intake and occurrence of kidney diseases.

3) Finally, a special session was organised in collaboration with EASO (European Association of the Study of Obesity) in order to celebrate the launch of the first meeting of the Healthy Hydration Working Group (HHWG). The topic of hydration status and obesity risk and prevention was considered with a special attention for childhood overweight and obesity.

\section{Definition of Hydration and Optimal Hydration Biomarkers}

Water is essential for all biological processes, and survival is largely linked to the ability to regulate total body water volume during periods of limitations of water accessibility. In the general population, the long-term effects of a low total body water turnover may have detrimental effects. In their article 'From state to process: defining hydration', Perrier et al. [8] show that the normal hydration state can be maintained despite large differences in the hydration process. The authors developed the concepts of hydration as a state or as a process and demonstrated the health importance of hydration state versus hydration process. Mounting evidence suggests that the hydration process may be more important than the hydration state in the context of long-term health. Hydration assessment using biomarkers of the hydration process is an important challenge. Munoz and colleagues are working in a laboratory which contributed to the quest for optimal hydration biomarkers. In their article 'Optimal hydration biomarkers: consideration of daily activities' [9], they discussed two main scenarios to optimise selection of hydration biomarkers. When selection of hydration biomarkers is requested, the results of the investigations reported in this article focus upon the need to consider previous and concurrent activities of patients and the frequency of sample measurements.

\section{Overview of the Relationships between Water Intake and Occurrence of Kidney Diseases}

The mammalian kidney is adapted to water reabsorption and to produce concentrated urine. The benefit of water in the prevention of kidney stones has been appreciated for decades while more recently a 'therapeutic' role of water was also suspected in the prevention of chronic kidney diseases. It is recognised that the hormone vasopressin (VP or AVP), which 
is normally suppressed by an increased fluid intake, could contribute to a progression toward chronic kidney diseases. By allowing water reabsorption, VP plays a crucial role in the ability of the kidney to concentrate urine. Deleterious effects of VP operate via disturbances induced in renal haemodynamics and blood pressure changes. Plasma VP levels are increased in patients with diabetic and non-diabetic kidney diseases. In animal models, suppression of VP by increased hydration or a VP receptor antagonist was associated with a reduced progression renal disease in models of kidney injury.

In the article 'Hydration and kidney health', Bouby et al. [10] highlight various aspects of the relationship between the renal status and hydration. The first section by Ronan Roussel, Christopher Taveau and Nadine Bouby deals with 'AVP, copeptin and renal function in diabetes'. The authors insist on the necessity for an identification of patients with type 2 diabetes and proteinuria at risk of developing end-stage renal disease. Copeptin, the stable C-terminal portion of VP, is an easy surrogate marker of VP and could be a candidate for the identification of patients at high risk of progression of nephropathy. The analysis of a large prospective cohort of patients with albuminuria and type 2 diabetes confirms that VP is possibly a risk factor for the decline of renal functions. In the second section, Connie J. Wang deals with 'Water as therapy in kidney diseases' and summarises the current state of knowledge regarding the potential beneficial role of water intake in nephrolithiasis, chronic kidney diseases and autosomal dominant polycystic kidney disease (ADPKD). With regard to the reported observations, the author is confident that the therapeutic benefits of water in humans with chronic kidney diseases or ADPKD will be confirmed in future randomised controlled trials. In the third section entitled 'Hydration in chronic kidney disease: observational studies lead to a randomized control trial', William F. Clark provides a critical overview of clinical studies and prior results of a pilot study preceding a large randomised control trial initiated in June 2013 that includes more than 700 participants with chronic kidney disease.

\section{Hydration and Obesity Prevention: The Launch of the EASO Healthy Hydration Working Group}

The EASO HHWG including obesity as well as hydration specialists was launched. The following topics have been addressed by the EASO HHWG:

- The consumption of various caloric sweeteners has increased markedly since the mid-20th century. A parallel with the rising prevalence of overweight and obesity has been noted world-wide.

- Epidemiological data and experimental studies have shown a positive correlation between sugar, fructose or SSB consumption and metabolic or obesity risks in children.

- As this correlation is still controversially discussed, repeated interventional and mechanistic studies are necessary the results of which must be communicated to the obesity research community.

The EASO HHWG has defined the following main objectives for its future research:

- To summarise the state of the science on fluid intake, overweight and obesity.

- To clarify the contribution of fluids to the overall energy intake and the epidemic of obesity.

- To identify and define knowledge gaps.

- To create a forum for scientific debate via conferences and workshops.

- To elaborate guidelines and promote education actions.

The session of the EASO HHWG at the 5th Annual Scientific Conference of the Hydration for Health began with a talk of Gema Frühbeck, President of EASO, and was followed by a talk of Volkan Yumuk, Chair of the Obesity Management Task Force of the EASO, who both provided a global overview of obesity-related questions. In their joint contribution 'Obesity: a gateway 
Lafontan et al.: $\mathrm{H} 4 \mathrm{H}-\mathrm{Hydration}$ for Health

disease with a rising prevalence' to the article 'Better hydration practices for obesity prevention?' [11], both speakers insist upon the silent menace of the obesity epidemic which becomes a world-wide health problem. They argue for a need of a true interdisciplinary research to address the questions raised by biological and psychological inter-individual variability in the obese populations in a given country and of the world. The obesity epidemic is reversible. It is considered that possibilities exist to reverse the trend and bring the epidemic under control.

The last article of this issue deals with 'Hydration and Obesity Prevention' [12]. In the first section 'Sugar-sweetened beverages and childhood obesity: the evidence calls for policy interventions', Janne C. de Ruyter and Jacob C. Seidell report the results of an intervention study they have performed for reducing SSB consumption. They noted that masked replacement of SSBs with non-caloric beverages reduced weight gain and fat accumulation in normal-weight children. Further results of community-based interventions in different parts of the world aiming at the consumption of sugar-free drinks are expected. The necessity and importance of a multi-factorial approach in the prevention of childhood obesity is becoming obvious for an increasing number of members of the community involved in the prevention and treatment. Childhood obesity prevention programmes have been launched in different countries. In the second section entitled 'EPODE, a multi-stakeholder approach to prevent childhood obesity' Jean-Michel Borys and colleagues detail the multi-stakeholder approach EPODE. Primary EPODE target groups are children ( 0 to 12 years old) and their families and all the local stakeholders who can influence childhood settings. Food consumption and physical activity management together with a better understanding of socio-cultural environments are concerned in the promotion of healthy active behaviours in children.

To conclude, the articles of this supplement provide directions for evaluation of subtle changes in hydration balance and for an optimised selection of hydration biomarkers. The impact of dehydration on kidney damage and the benefits of water intake for kidney health have been discussed. However, randomised controlled trials including larger populations are still needed to elucidate the impact of healthy hydration on renal physiology and pathology. Concerning obesity-related questions and more specifically childhood obesity primary prevention, the articles presented here pinpoint the necessity of public health measures to reduce SSB consumption and propose healthy hydration procedures aiming at increased water intake. Primary and secondary intervention studies evaluating the impact of a management of fluid intake must be expanded in different countries. Moreover, children caregivers must be educated about the health benefits of water consumption. In addition, actors of the youth health-care system as well as parents and teachers must increase water availability in day-care settings. Expansion of global strategies such as the EPODE methodology will certainly facilitate initiatives in other countries [13].

\section{Disclosure Statement}

ML is an occasional consultant for Danone Nutricia Research.

\section{References}

1 Daniels MC, Popkin BM: Impact of water intake on energy intake and weight status: a systematic review. Nutr Rev 2010;68:505-521.

2 Du SF, Wang HJ, Zhang B, Zhai FY, Popkin BM: China in the period of transition from scarcity and extensive undernutrition to emerging nutrition-related non-communicable diseases, 1949-1992. Obes Rev 2014; 15(suppl 1):8-15.

-3 Shirreffs SM: Global patterns of water intake: how intake data affect recommendations. Nutr Rev 2012; 70(suppl 2):S98-100. 
4 Bray GA, Popkin BM: Calorie-sweetened beverages and fructose: what have we learned 10 years later. Pediatr Obes 2013;8:242-248.

5 Hu FB: Resolved: there is sufficient scientific evidence that decreasing sugar-sweetened beverage consumption will reduce the prevalence of obesity and obesity-related diseases. Obes Rev 2013;14:606-619.

6 Armstrong LE, Braquera S, Duhamel J-F, Hardinsyah R, Haslam D, Lafontan M: Recommendations for healthier hydration: addressing the public health issues of obesity and type 2 diabetes. Clin Obes 2013;2:115-124.

-7 Lotan Y, Buendia Jimenez I, Lenoir-Wijnkoop I, Daudon M, Molinier L, Tack I, Nuijten MJ: Increased water intake as a prevention strategy for recurrent urolithiasis: major impact of compliance on cost-effectiveness. J Urol 2013;189:935-939.

8 Perrier ET, Armstrong LE, Daudon M, Kavouras S, Lafontan M, Lang F, Péronnet F, Stookey JD, Tack I, Klein A: From state to process: defining hydration. Obes Facts 2014;7(suppl 2): DOI: 10.1159/000360611.

-9 Muñoz CX, McKenzie AL, Armstrong LE: Optimal hydration biomarkers: consideration of daily activities. Obes Facts 2014;7(suppl 2): DOI: 10.1159/000360655.

$\checkmark 10$ Bouby N, Clark WC, Roussel R, Taveau C, Wang CJ: Hydration and kidney health. Obes Facts 2014;7(suppl 2): DOI: $10.1159 / 000360889$.

11 Frühbeck G, Yumuk V: Obesity: a gateway disease with a rising prevalence. Obes Facts 2014;7(suppl 2): DOI: $10.1159 / 000361004$.

12 Borys J-M, de Ruyter JC, Finch H, Harper P, Levy E, Mayer J, Richard P, Ruault du Plessis H, Seidell JC, Vinck J: Hydration and obesity prevention. Obes Facts 2014;7(suppl 2): DOI: 10.1159/000360748.

13 EPODE European Network: www.epode-european-network.com (last accessed February 18, 2014). 